\title{
Progress of the SI and CGS Systems: Conversion of the MKSA Units to the MKS and CGS Units
}

\author{
Askar Abdukadyrov \\ Mechanical Faculty, Kyrgyz Technical University, Bishkek, Kyrgyzstan \\ Email address: \\ abdukadyrov64@mail.ru \\ To cite this article: \\ Askar Abdukadyrov. Progress of the SI and CGS Systems: Conversion of the MKSA Units to the MKS and CGS Units. American Journal of \\ Electromagnetics and Applications. Vol. 6, No. 1, 2018, pp. 24-27. doi: 10.11648/j.ajea.20180601.14
}

Received: April 16, 2018; Accepted: May 2, 2018; Published: May 22, 2018

\begin{abstract}
Recording of the equations in mechanics does not depend on the choice of system of measurement. However, in electrodynamics the dimension of the electromagnetic quantities (involving current, charge and so on) depends on the choice of unit systems, such as SI (MKSA), CGSE, CGSM, Gaussian system. We show that the units of the MKSA system can be written in the units of the MKS system and in conforming units of the CGS system. This conversion allows to unify the formulas for the laws of electromagnetism in SI and CGS. Using the conversion of units we received two new formulas that complement Maxwell's equations and allow deeper to understand the nature of electromagnetic phenomena, in particular, the mechanism of propagation of electromagnetic waves.
\end{abstract}

Keywords: SI, CGS, Unit Systems, Conversion of Units, Maxwell's Equations

\section{Introduction}

The International System of Units (SI) and the $\mathrm{cm}-\mathrm{g}-\mathrm{S}$ system of units (CGS) are variants of the metric system of physical units. The equations for laws of mechanics are not affected by the choice of units. However, in electrodynamics a situation is different: the formulas for physical laws of electromagnetism (such as Maxwell's equations) are depended by a choice of the unit system.

Within CGS, there are several different unit "sub-systems", including the CGS system of electrostatic units (CGSE), the CGS system of electromagnetic units (CGSM) and Gaussian system of units (is a kind of unification of the CGSE and CGSM systems) [1]. The electromagnetic quantities (involving current, charge and so on) have different numerical value and dimension in the different systems of units. For instance, the elementary electric charge (denoted as e)

in the CGSE system $e_{E}=4.8 \times 10^{-10} \mathrm{~g}^{1 / 2} \mathrm{~cm}^{3 / 2} \mathrm{~s}^{-1}$,

in the CGSM system $e_{M}=1.6 \times 10^{-20} \mathrm{~g}^{1 / 2} \mathrm{~cm}^{1 / 2}$.

The SI is a composite system: it includes, in particular, the $m-k g-s$ system of mechanical units (MKS) and the $m-k g-s-A$ system of electromagnetic units (MKSA). Therefore, speaking about the charge in the SI need to indicate the MKSA sub-system: in the MKSA $e=1.6 \times 10^{-19} \mathrm{C}$.
Thus, to unify the formulas of electrodynamics in SI and CGS, need to convert the MKSA units in the MKS units and in conforming CGS units, as in the case of mechanical units, such as newton $\left(\mathrm{N}=\mathrm{kg} \mathrm{m} \mathrm{s}^{-2}=10^{5} \mathrm{~g} \mathrm{~cm} \mathrm{~s}^{-2}\right)$, joule $(\mathrm{J}=\mathrm{N} \mathrm{m}$ $=\mathrm{kg} \mathrm{m}^{2} \mathrm{~s}^{-2}=10^{7} \mathrm{~g} \mathrm{~cm}^{2} \mathrm{~s}^{-2}$ ) and so on.

\section{Method}

First of all, need to determine the correct dimension of the electric charge in the MKS and CGS systems. To do this, will consider two examples.

Example 1. There is the known formula

$$
r_{0}=k e^{2} / m c^{2}
$$

where $r_{0}$ is so-called "classical radius of the electron", $k$ is the coefficient of proportionality, $m$ is the mass of the electron, $c$ $=3 \times 10^{8} \mathrm{~m} \mathrm{~s}^{-1}=3 \times 10^{10} \mathrm{~cm} \mathrm{~s}^{-1}$ is the speed of light in vacuum. From this equation it follows:

$$
k e^{2}=r_{0} m c^{2} .
$$

It should be noted that only having mass particles also have an electric charge, i.e. massless particles have no charge. In other words, there is a connection between the charge of an elementary particle and its mass. Therefore 


$$
\left[k e^{2}\right]=\left[r_{0} m c^{2}\right]=\left[\left(r_{0} / m\right)(m c)^{2}\right] .
$$

Hence are easy to find the dimension of the coefficient $k$ and the charge $e$ in the MKS and CGS systems:

$$
\begin{gathered}
{[k]=\left[r_{0} / m\right]=\mathrm{m} \mathrm{kg}^{-1}\left({\text { or } \left.\mathrm{cm} \mathrm{g}^{-1}\right),},\right.} \\
{[e]=[m c]=\mathrm{kg} \mathrm{m} \mathrm{s}^{-1}\left(\text { or } \mathrm{g} \mathrm{cm} \mathrm{s}^{-1}\right) .}
\end{gathered}
$$

Example 2. According to the hydrogen atom model proposed by N. Bohr in 1913 [2], the electron moves with a speed $v$ around the nucleus in circular orbit of radius $r$. The force of the interaction of the electron with the nucleus $k e^{2} / r^{2}$ gives to the electron of mass $m$ the centripetal acceleration $v^{2} / r$ :

$$
k e^{2} / r^{2}=m v^{2} / r
$$

Hence

$$
k e^{2}=r m v^{2}
$$

and

$$
\left[k e^{2}\right]=\left[r m v^{2}\right]=\left[(r / m)(m v)^{2}\right] .
$$

It's obvious that

$$
\begin{gathered}
{[k]=[r / m]=\mathrm{m} \mathrm{kg}^{-1}\left(\text { or } \mathrm{cm} \mathrm{g}^{-1}\right),} \\
{[e]=[m v]=\mathrm{kg} \mathrm{m} \mathrm{s}^{-1}\left(\text { or } \mathrm{g} \mathrm{cm} \mathrm{s}^{-1}\right) .}
\end{gathered}
$$

So we have determined that in the MKS and CGS systems the charge has the dimension of momentum.

To determine the numerical values of the charge in units of the MKS and CGS is need to use two fundamental laws.

According to Coulomb's law the absolute value of the force of electrostatic interaction between two point charges in a vacuum in the SI

$$
F=k q_{1} q_{2} / r^{2},
$$

where $k=9 \times 10^{9} \mathrm{~N} \mathrm{~m}^{2} \mathrm{C}^{-2}\left(\mathrm{~kg} \mathrm{~m}^{3} \mathrm{~s}^{-2} \mathrm{C}^{-2}\right)$ is a proportionality factor, $q_{1}$ and $q_{2}$ are the quantities of charges in coulombs, $r$ is the distance between them. This law can be rewritten as:

$$
F=q_{1} k^{1 / 2} q_{2} k^{1 / 2} / r^{2}=Q_{1} Q_{2} / r^{2},
$$

where $Q=q k^{1 / 2}$ is charge in the CGSE units. It turns out that mathematically the charge $Q$ (in electrostatic units, esu) is the product of the charge $q$ (in coulombs) and $k^{1 / 2}$. For instance,

$$
1 \mathrm{C} \times k^{1 / 2}=3 \times 10^{9 / 2} \mathrm{~kg}^{1 / 2} \mathrm{~m}^{3 / 2} \mathrm{~s}^{-1}=3 \times 10^{9} \mathrm{~g}^{1 / 2} \mathrm{~cm}^{3 / 2} \mathrm{~s}^{-1} .
$$

According to Ampere's law the absolute value of the force of magnetic interaction between two infinitely thin parallel current-carrying conductors in a vacuum in the SI

$$
F=2 k_{M} i_{1} i_{2} l / r
$$

where $k_{M}=10^{-7} \mathrm{~N} \mathrm{~A}^{-2}\left(\mathrm{~kg} \mathrm{~m} \mathrm{~s}^{-2} \mathrm{~A}^{-2}\right)$ is a proportionality factor, $i_{1}$ and $i_{2}$ are the values of electric currents in amperes, $l$ is length of the conductors, $r$ is distance between them. This law can be rewritten as:

$$
F=2 i_{1} k_{M}{ }^{1 / 2} i_{2} k_{M}{ }^{1 / 2} l / r=2 I_{1} I_{2} l / r,
$$

where $I=i k_{M}{ }^{1 / 2}$ is current in the CGSM units. It turns out that mathematically the current $I$ (in the CGSM units) is the product of current $i$ (in amperes) and $k_{M}{ }^{1 / 2}$. For instance,

$$
\begin{gathered}
1 \mathrm{~A} \times k_{M}^{1 / 2}=10^{-7 / 2} \mathrm{~kg}^{1 / 2} \mathrm{~m}^{1 / 2} \mathrm{~s}^{-1} \\
\text { dyne }^{1 / 2} \text { ). }
\end{gathered}=0.1 \mathrm{~g}^{1 / 2} \mathrm{~cm}^{1 / 2} \mathrm{~s}^{-1} \text { (or } 0.1
$$

Therefore it is considered that the ampere is 0.1 of the CGSM unit of current.

Charge $Q_{M}$ (in the CGSM units) is defined as current $I$ multiplied by time $t$ (in seconds):

$$
Q_{M}=I t=i k_{M}{ }^{1 / 2} t=q k_{M}{ }^{1 / 2}(q=i t) .
$$

That is, mathematically the charge $Q_{M}$ (in electromagnetic units, emu) is the product of the charge $q$ (in coulombs) and $k_{M}^{1 / 2}$. For instance,

$$
1 \mathrm{C} \times k_{M}^{1 / 2}=10^{-7 / 2} \mathrm{~kg}^{1 / 2} \mathrm{~m}^{1 / 2}=0.1 \mathrm{~g}^{1 / 2} \mathrm{~cm}^{1 / 2} .
$$

Therefore it is considered that the coulomb is 0.1 of the CGSM unit of charge.

By definition [3]: "The ampere is that constant current which, if maintained in two straight parallel conductors of infinite length, of negligible circular cross-section, and placed 1 metre apart in vacuum, would produce between these conductors a force equal to $2 \times 10^{-7}$ newton per metre of length".

Since ampere is defined via a force $(F)$ existing between two currents, the dimension of ampere $[\mathrm{A}]$ is equal to the dimension of force $[F]$, which is measured in the SI in newtons $(\mathrm{N})$ :

$$
[\mathrm{A}]=[F]=\mathrm{N} \text {. }
$$

Therefore, the dimension of proportionality factor $k_{M}$ is inverse to the dimension of force:

$$
\left[k_{M}\right]=\mathrm{N}[\mathrm{A}]^{-2}=\mathrm{N}^{-1}=[F]^{-1} .
$$

Consequently, coefficient $k_{M}=1 / F_{1}$, where $F_{1}$ is the unit of force.

By definition, the CGSM unit of current is that constant current which, if maintained in two straight parallel conductors of infinite length, of negligible circular crosssection, and placed $1 \mathrm{~cm}$ apart in a vacuum, would produce between these conductors a force equal to 2 dyne per $1 \mathrm{~cm}$ of length.

Digit 2 is included in Ampere's law, so the force $F_{1}=1$ dyn $=1 \mathrm{~g} \mathrm{~cm} \mathrm{~s}^{-2}$ (equal to $10^{-5} \mathrm{~N}$ ) is the CGS system unit of force. Hence, we find [4]:

$$
\begin{gathered}
\mathrm{A}=0.1 F_{1}=10^{-6} \mathrm{~N}=0.1 \text { dyn, } \\
\mathrm{C}=\mathrm{A} \cdot \mathrm{s}=10^{-6} \mathrm{~N} \mathrm{~s}=0.1 \text { dyn } \mathrm{s}, \\
k_{M}=10^{-7} \mathrm{~N} \mathrm{~A}^{-2}=1 /\left(10^{-5} \mathrm{~N}\right)=1 /(1 \mathrm{dyn}) .
\end{gathered}
$$

It should be recalled, in 1856 German scientists W. Weber and R. Kohlrausch experimentally determined that the ratio 
of the charge in electrostatic units $(Q)$ to the same charge in electromagnetic units $\left(Q_{M}\right)$ is numerically equal to the speed of light in vacuum $(c)[5,6]$ :

$$
Q / Q_{M}=k^{1 / 2} / k_{M}^{1 / 2}=c .
$$

Therefore,

$$
k=k_{M} \cdot c^{2}=c^{2} /\left(10^{-5} \mathrm{~N}\right)=c^{2} /(1 \mathrm{dyn}) .
$$

A numeric value of the coefficient $k$ in the MKS and CGS units is equal to:

$$
k=9 \times 10^{9} \mathrm{~N} \mathrm{~m}^{2} \mathrm{C}^{-2}=9 \times 10^{21} \mathrm{~m} \mathrm{~kg}^{-1}=9 \times 10^{20} \mathrm{cmg}^{-1} .
$$

The charge $e$ in the MKSA, MKS and CGS units we will call the true elementary charge:

$$
e=1.6 \times 10^{-19} \mathrm{C}=1.6 \times 10^{-25} \mathrm{~kg} \mathrm{~m} \mathrm{~s}^{-1}=1.6 \times 10^{-20} \mathrm{~g} \mathrm{~cm} \mathrm{~s}^{-1} \text {. }
$$

It is obvious that mathematically the CGSE system elementary charge $e_{E}$ is product of the true charge $e$ and $k^{1 / 2}$, the CGSM system elementary charge $e_{M}$ is product of the true charge $e$ and $k_{M}^{1 / 2}$ :

$$
\begin{gathered}
e_{E}=e k^{1 / 2}=e c /(1 \mathrm{dyn})^{1 / 2}=4.8 \times 10^{-10} \mathrm{esu}, \\
e_{M}=e k_{M}^{1 / 2}=e /(1 \mathrm{dyn})^{1 / 2}=1.6 \times 10^{-20} \mathrm{emu} .
\end{gathered}
$$

Now we can convert the MKSA units to the MKS and CGS units.

\section{Conversion of the MKSA Units to the MKS and CGS Units}

Electric current $(I) \mathrm{A}=10^{-6} \mathrm{~kg} \mathrm{~m} \mathrm{~s}^{-2}=0.1 \mathrm{~g} \mathrm{~cm} \mathrm{~s}^{-2}$; Electric charge $(Q) \mathrm{C}=\mathrm{A} \cdot \mathrm{s}=10^{-6} \mathrm{~kg} \mathrm{~m} \mathrm{~s}^{-1}=0.1 \mathrm{~g} \mathrm{~cm} \mathrm{~s}^{-1}$; Potential difference $(\varphi) \mathrm{V}=\mathrm{J} / \mathrm{C}=10^{6} \mathrm{~m} \mathrm{~s}^{-1}=10^{8} \mathrm{~cm} \mathrm{~s}^{-1}$; Electric capacitance $(C) \mathrm{F}=\mathrm{C} / \mathrm{V}=10^{-12} \mathrm{~kg}=10^{-9} \mathrm{~g}$;

Electrical resistance $(R) \Omega=\mathrm{V} / \mathrm{A}=10^{12} \mathrm{~s} \mathrm{~kg}^{-1}=10^{9} \mathrm{~s} \mathrm{~g}^{-1}$; Electrical conductance $(G) \mathrm{S}=\mathrm{A} / \mathrm{V}=10^{-12} \mathrm{~kg} \mathrm{~s}^{-1}=10^{-9} \mathrm{~g} \mathrm{~s}^{-1}$; Electric field strength $(E) \mathrm{N} / \mathrm{C}=\mathrm{V} / \mathrm{m}=10^{6} \mathrm{~s}^{-1}$;

Electric displacement $(D) \mathrm{C} / \mathrm{m}^{2}=10^{-6} \mathrm{~kg} \mathrm{~m}^{-1} \mathrm{~s}^{-1}=10^{-5} \mathrm{~g} \mathrm{~cm}^{-1} \mathrm{~s}^{-1}$; Magnetic flux density $(\Phi) \mathrm{Wb}=\mathrm{V} \cdot \mathrm{c}=10^{6} \mathrm{~m}=10^{8} \mathrm{~cm}$;

Magnetic field strength $(H) \mathrm{A} / \mathrm{m}=10^{-6} \mathrm{~kg} \mathrm{~s}^{-2}=10^{-3} \mathrm{~g} \mathrm{~s}^{-2}$; Magnetic field induction $(B) \mathrm{T}=\mathrm{Wb} / \mathrm{m}^{2}=10^{6} \mathrm{~m}^{-1}=10^{4} \mathrm{~cm}^{-1}$; Inductance $(L) \mathrm{H}=\mathrm{Wb} / \mathrm{A}=10^{12} \mathrm{~s}^{2} \mathrm{~kg}^{-1}=10^{9} \mathrm{~s}^{2} \mathrm{~g}^{-1}$.

\section{Example of Unit Conversion Application}

The basis of the classical electrodynamics are the Maxwell's equations in which are use vector values of intensity and induction: electrical $\boldsymbol{E}, \boldsymbol{D}$ and magnetic $\boldsymbol{H}, \boldsymbol{B}$. For example, in the absence of charges and currents this equations for the vacuum in the SI system have the form:

$\varepsilon_{0}(\partial \boldsymbol{E} / \partial t)=\operatorname{rot} \boldsymbol{H}, \mu_{0}(\partial \boldsymbol{H} / \partial t)=-\operatorname{rot} \boldsymbol{E}, \operatorname{div} \boldsymbol{E}=0, \operatorname{div} \boldsymbol{H}=0,(30)$

where $\varepsilon_{0}$ and $\mu_{0}$ are the electric and magnetic constants, $\varepsilon_{0} \boldsymbol{E}=$
$\boldsymbol{D}, \mu_{0} \boldsymbol{H}=\boldsymbol{B}[7,8]$.

On the basis of own equations J. Maxwell predicted the existence of electromagnetic waves. However, the mechanism of propagation of electromagnetic waves is still unclear.

A basic property of all waves, whatever their nature, is the transfer of energy without transfer of matter. Light is a kind of electromagnetic waves. Initially it was assumed that light waves propagate in a special light-bearing medium - ether, filling all the world space. After as in beginning of the twentieth century the concept of ether was rejected, had no choice as to admit that a medium for light propagation and, more generally, of electromagnetic waves is itself a threedimensional space.

In accordance with quantum theory, it is customary assumed that the space is consisted of the uncountable smallest parts (elements) of space. Denote by $r$ the displacement of the elements of the space in which electromagnetic waves propagate. Then expressions (30) allow two possibilities to consider one vector quantity as speed of displacement of elements of space $\partial r / \partial t$ (i.e. as translational motion), and another - as a rotor (vortex) of a vector of displacement of these elements rot $r$ (i.e. as rotational movement):

$$
\begin{aligned}
& \partial r / \partial t=\boldsymbol{E}, \operatorname{rot} r=-\boldsymbol{B} \\
& \partial r \partial t=\boldsymbol{H}, \operatorname{rot} r=\boldsymbol{D}
\end{aligned}
$$

The current Maxwell's theory cannot determine which from the two options is correct. In order to make an unambiguous choice, it is necessary to use the stated conversion of the MKSA units into the MKS units.

The SI unit of electric strength $(E) \mathrm{N} / \mathrm{C}=10^{6} \mathrm{~s}^{-1}$. Since the second (s) is a unit of time $(t)$, the electric strength makes sense of the frequency, i.e. inversely proportional to the time interval:

$$
E \sim 1 / t
$$

It is obvious that the first variant does not correspond to reality, because $\partial r / \partial t$ is a physical quantity and at $r=1$ the expression $\partial 1 / \partial t$ is simply meaningless. Remains the second option.

The SI unit of magnetic intensity $(H) \mathrm{A} / \mathrm{m}=10^{-6} \mathrm{~kg} / \mathrm{s}^{2}=$ $10^{6} \mathrm{~S} / \mathrm{s}$, where $\mathrm{S}=10^{-12} \mathrm{~kg} / \mathrm{s}$ - the unit of conductance $(G)$. Thus, the magnetic intensity is proportional to the conductance and inversely proportional to the time interval:

$$
H \sim G / t
$$

The SI unit of electric displacement $(D) \mathrm{C} / \mathrm{m}^{2}=10^{-6} \mathrm{~kg} /(\mathrm{s}$ $\mathrm{m})=10^{6} \mathrm{~S} / \mathrm{m}$, where $\mathrm{S} / \mathrm{m}=10^{-12} \mathrm{~kg} /(\mathrm{s} \mathrm{m})$ - the unit of electrical conductivity $(\sigma)$. Thus, the electric induction is proportional to the conductivity:

$$
D \sim \sigma
$$

Comparing dimensions $G$ and $\sigma$ and considering equations (34) and (35) we see that $r=G$ and the second variant 
uniquely does correspond to the physical reality:

$$
\partial r / \partial t=\boldsymbol{H}=\partial \boldsymbol{G} / \partial t, \operatorname{rot} r=\boldsymbol{D}=\operatorname{rot} \boldsymbol{G} .
$$

Thus, the magnetic intensity $(H)$ is determined by the rate of displacement of the elements of space (or the rate of change in the conductance of space), and the electric induction $(D)$ is determined by the rotation of the elements of space. Consequently, the conductance of space, i.e. the transfer of energy by electromagnetic waves from one element of space to another, is carried out by rotation of these elements.

Almost to the same conclusion came the Irish physicist G. FitzGerald in 1878, with the difference that he was talking about the elements of ether, not about the elements of space. The scientist wanted to use the "rotational" ether theory, was created by his countryman J. MacCullagh, to improve the Maxwell's theory of electromagnetism because in the part relating to mathematical formulas, the both theories are completely the same $[9,10]$.

\section{Conclusions}

We have proved that electromagnetic units of the MKSA system can be expressed in units of the MKS and CGS systems, as in the case of mechanical units (such as newton, joule and other). This result allows to unify the formulas of the electrodynamics in the SI and CGS, i.e. the formulas will be written the same way. Thus, there is the possibility of fully combining the electrodynamics and mechanics. It should be recalled that at classical level the mechanics is united with gravity. Therefore, the unification of the electrodynamics and mechanics is the step forward to the unification of electromagnetism with gravity what is necessary to build an Unified Theory.

\section{References}

[1] F. Cardarelli, Encyclopaedia of Scientific Units, Weights and Measures: Their SI Equivalences and Origins, (2nd ed., Springer, 2003) pp. 20-25.

[2] N. Bohr, Philosophical Magazine, 26 (153), 476-502 (1913).

[3] Bureau International des Poids et Mesures (BIPM), SI Brochure: The International System of Units (SI), (8th ed., 2006), http://www.bipm.org/en/publications/si-brochure/

[4] A. B. Abdukadyrov, Physics - unity in diversity, (Bishkek, 2005) pp. 12-15.

[5] W. Weber and R. Kohlrausch, Annalen der Physik und Chemie, 99, 10-25 (1856).

[6] J. F. Keithley, The story of electrical and magnetic measurements: from 500 B. C. to the 1940s, (New York: IEEE Press, 1999) p. 115.

[7] J. D. Jackson, Classical Electrodynamics, (3rd ed., New York: Wiley, 1999) pp. 2-4.

[8] D. J. Griffiths, Introduction to Electrodynamics, (4th ed., Cambridge University Press, 2017) pp. 332-337.

[9] B. J. Hunt, The Maxwellians, (Ithaca, New York: Cornell University Press, 2005) pp. 15-19.

[10] V. S. Kirsanov, "Ether and genesis of classical field theory", in: Mechanics and Civilization XVII-XIX Centuries, (Moscow: Nauka, 1979) pp. 255-258. 